\title{
Energy Efficient WSN with Cluster Head Rotation Algorithm for Underground Mine Supervising Environment
}

\author{
Archana K R $\mathbf{R}^{1}$, Kingsley $\mathbf{G}^{2}$ \\ PG Scholar, Department of ECE, CMS College of Engineering and Technology, Coimbatore, India ${ }^{1}$ \\ Assistant Professor, Department of ECE, CMS College of Engineering and Technology, Coimbatore, India ${ }^{2}$
}

\begin{abstract}
Underground mines are more hazardous in nature and always contain unsafe for their workers. Every year, underground mining causes huge losses due to accidents and disaster. There is the need to develop an effective, secure, reliable and low-cost surveillance and safety system in underground mine environments. Wireless Sensor Networks (WSNs) are increasingly being used for such application. Since wireless sensor nodes are highly energy constrained devices and they have limited battery life due to various constraints of sensor nodes such as size and cost, etc. Therefore, optimal usage of node energy is the major challenge in wireless sensor networks. Clustering of sensor nodes is an effective method to use the node energy optimally and prolong lifetime of such energy constrained wireless sensor networks. In this paper, we propose an energy efficient wireless sensor node with cluster head rotation algorithm for supervising the underground mine environment. Proposed clustering algorithm ensures balanced size cluster formation and it has been proposed to achieve balanced energy consumption among nodes.
\end{abstract}

Keywords: Wireless Sensor Networks (WSNs), Clusters, Cluster head, and Underground mines

\section{INTRODUCTION}

Mining is the process or industry of obtaining coal or other minerals from a mine. The activities carried out in order to obtain these minerals have dangerous element to them. Although technological advancements and strict regulations have helped in controlling the accident rate in underground mine environment, still hundreds of people lose their lives and huge financial losses are incurred to mining industry every year from mining disasters. Most of accidents occurred in underground mines are landslides, water breakage gas explosion, fire explosion, roof collapses and suffocations etc. These accidents and disasters pose serious problem of managing safety and health risks to mine workers. As such, mining industry constantly looking for new methods to increase workers safety, increase production volume and reduce operating cost. Safety in underground mine environment requires continuous monitoring of parameters such as temperature, humidity, oxygen level and some noxious gases (i.e. $\mathrm{CH} 4, \mathrm{CO}, \mathrm{CO} 2$ and others) that are produced during various mining operations. Recent advancements in large scale integration and wireless communication technologies have enabled the development of small size, low cost, and multi-functional devices known as sensor nodes. Sensor nodes are capable of sensing the desired environmental parameters within their vicinity, such as temperature, pressure, humidity and pollutants, etc., converting the sensed variable to electrical signal and transmitting the sensed data to the desired destination. To achieve these objectives, sensor nodes are equipped with sensors, microcontrollers / microprocessors, and wireless trans-receivers.

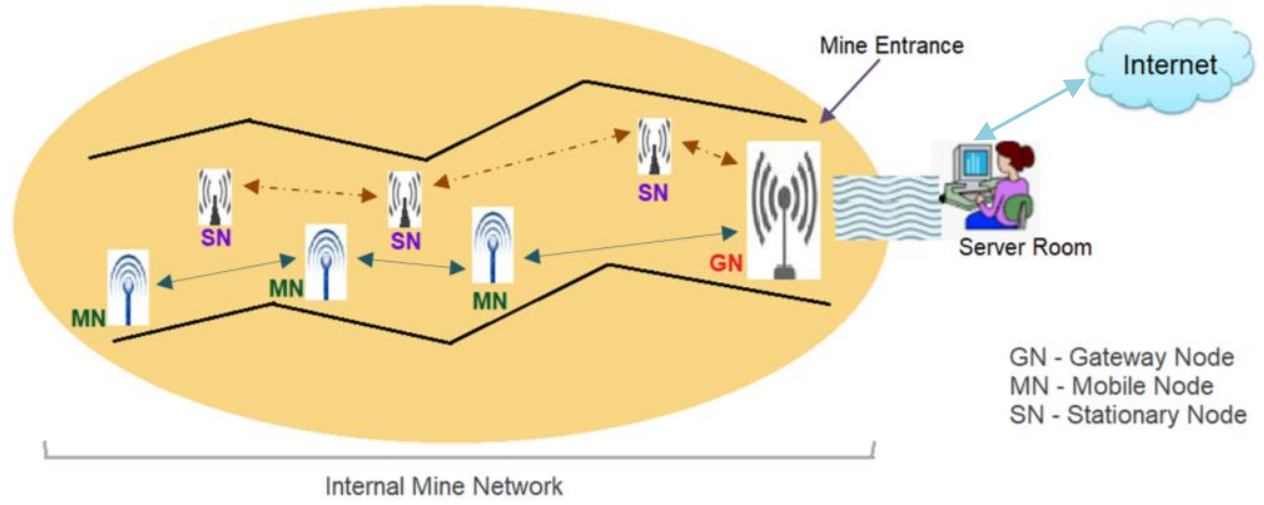

Fig.1. Overall System Architecture 
There is always the need to locate and communicate with miners, particularly in the event of fires, roof falls or other life-threatening situations. Safety in the working condition encourages for more productivity and self confidence among the workers.

A wireless system is not a single device, but a collection of devices which operate interdependently. Wireless sensor network is being composed of a large number of sensors. Due to deployment of wireless sensors in unattended harsh environment, it is not possible to charge or replace their batteries. Therefore, energy efficient operation of wireless sensors to prolong the lifetime of overall wireless sensor network is of utmost importance. Proposed energy efficient wireless underground mine environment monitoring system is developed using cluster head rotation algorithm.

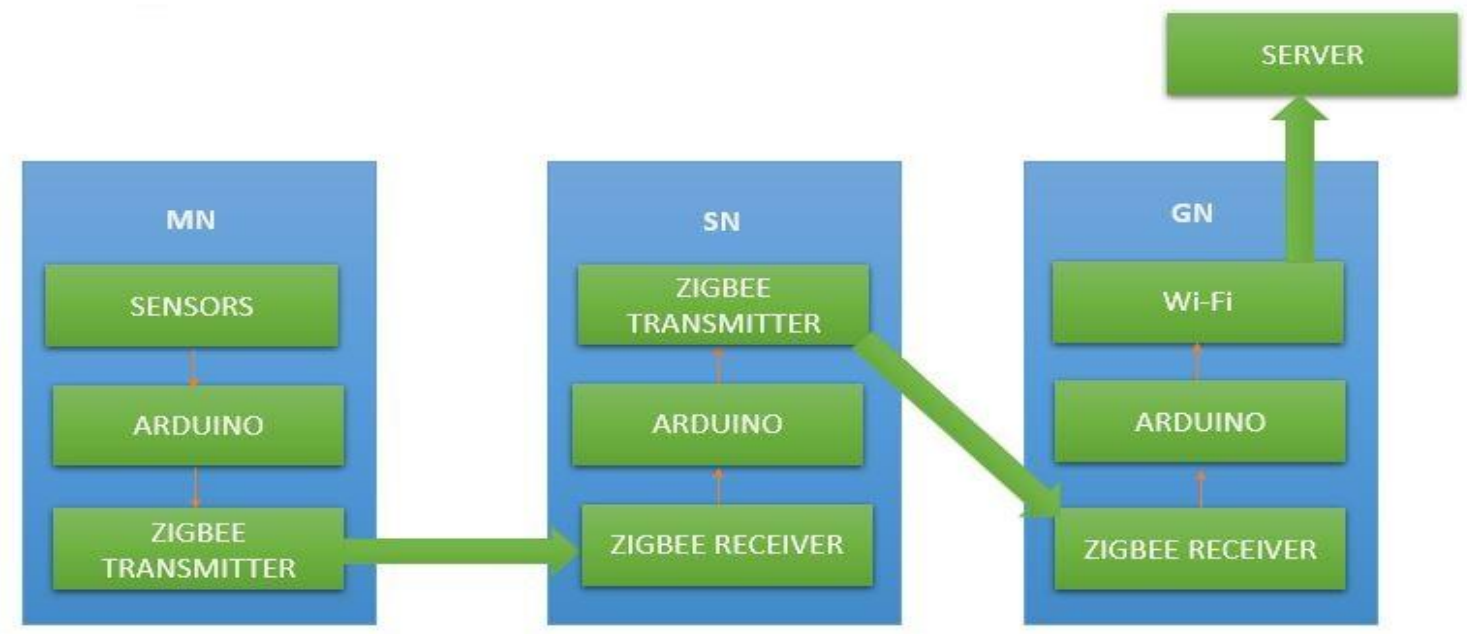

Fig.2. Block diagram of WSN monitoring system

\section{PROPOSED SYSTEM}

Typical underground mine consists of stretches of kilometers long tunnels with excavation branches spreading out like a tree. Underground mine environment requires continuous monitoring of parameters. So, the proposed system consists of a wireless underground mine environment monitoring system and cluster head rotation algorithm.

\section{A. WSN Monitoring System}

A Wireless Sensor Network is a self-organized wireless network composed of a large number of sensor nodes that interact with the physical world. These bunch of nodes are placed at appropriate positions such that users can conveniently know the environment parameters. Each type of device runs its specific firmware and communicate, via set communication protocol. The function of this network is to collect the data from the environment and send it to the sink node. The components of WSN are; sensor nodes, base station and, routers etc. It consists of a large number of sensor nodes that collect information from the environment and transmit to the gateway node. The WSN monitoring system consists of
- $\quad$ Mobile node
- Stationary node
- $\quad$ Gateway node

\section{Mobile node:}

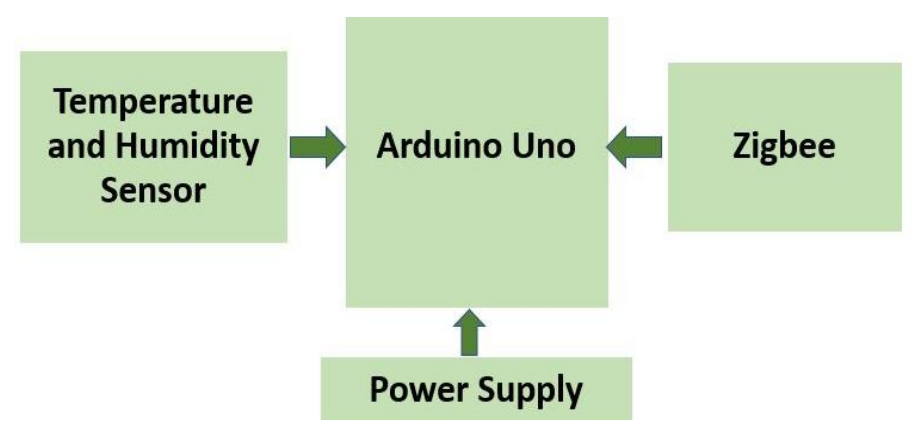

Fig.3. Block diagram of MN node 
Vol. 8, Issue 4, April 2019

MNs are carried by the miners. The nodes are capable of monitoring miner activity, sense parameters critical for miner's survival inside the mine (e.g. temperature, humidity, and oxygen levels) and convey information to the nearest SN. Miners can also send distress signal(s) to the gateway as well as run miner localization algorithm. Here both zigbee and temperature sensor is interfaced with arduino with appropriate embedded c program and connections. The nodes are capable of monitoring miner activity, sense parameters critical for miner's survival inside the mine (e.g. temperature, humidity, and oxygen levels) and convey information to the nearest SN. Miners can also send distress signal(s) to the gateway as well as run miner localization algorithm.

Stationary node: SNs are deployed throughout the mine at an appropriate distance from each other. SNs sense different parameters critical to structural integrity of the mine (e.g. roof fall, temperature, concentration of toxic gases such as carbon monoxide (CO), methane). Based on readings of different attributes, SNs run local event detection and identification algorithm. This distributed processing is one of the key features of the proposed system. This means that in case a node or certain number of nodes are disconnected from server, they can still use the gathered data (of that particular region) for localized event detection and localized alarm generation.

Here both zigbee, gas sensor and temperature sensor is interfaced with arduino with appropriate embedded c program and connections. SNs sense different parameters critical to structural integrity of the mine (e.g. roof fall, temperature, humidity, and concentration of toxic gases such as carbon monoxide (CO)). Based on readings of different attributes, SNs run local event detection and identification algorithm.

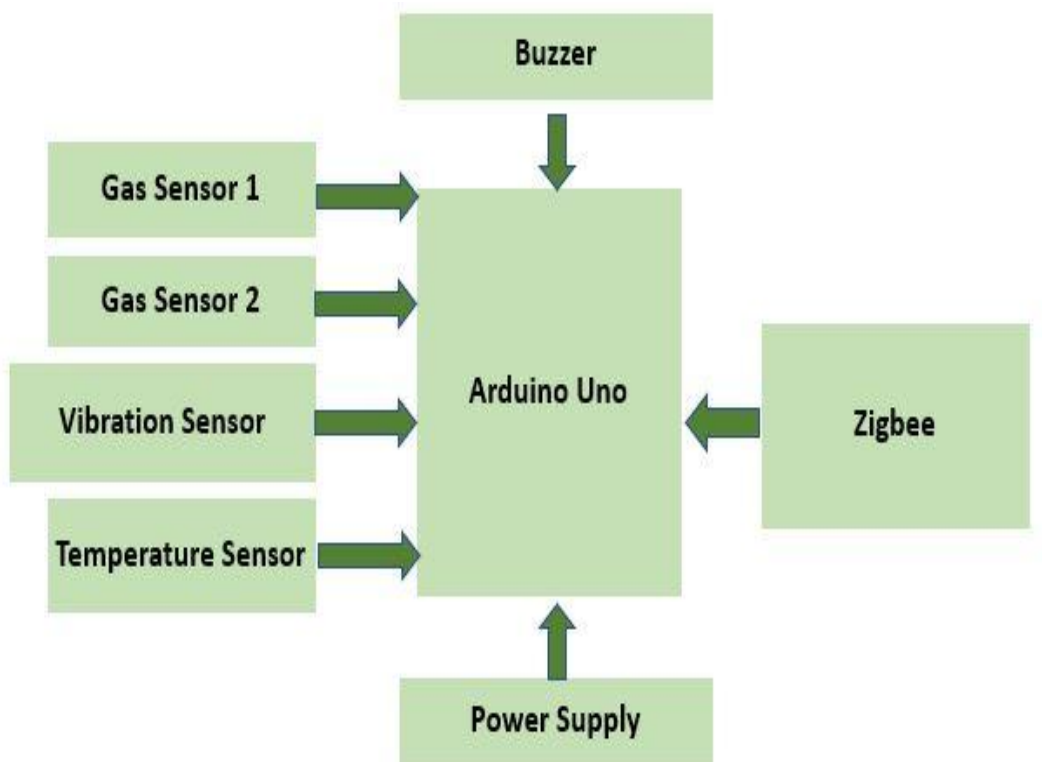

Fig.4. Block diagram of SN node

Gateway node: GN collects data from SNs in its vicinity using custom designed protocol and sends it to the server/BS via Ethernet or Wi-Fi. At BS, data received from GN can be visually analyzed. BS is also responsible for declaring a global event. Proposed system also supports down-link communication from the BS to one or multiple SNs. Here both zigbee and wifi module is interfaced with arduino with appropriate embedded c program and connections. GN collects data from SNs in its vicinity using custom designed protocol and sends it to the server/BS via Ethernet or Wi-Fi. At BS, data received from GN can be visually analyzed. BS is also responsible for declaring a global event. GN collects data from SNs in its vicinity using custom designed protocol and sends it to the server/BS via Ethernet or Wi-Fi. At BS, data received from GN can be visually analyzed. BS is also responsible for declaring a global event.

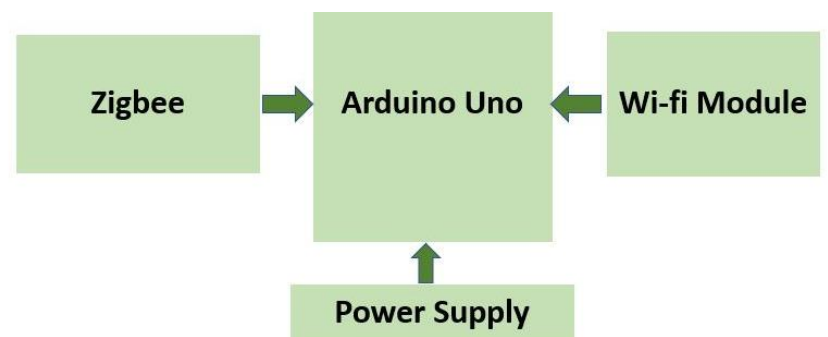




\section{B. Cluster Head Rotation}

The proposed model is a cluster based three tier-architectures where first tier records the first-run configuration of the nodes. The second tier secures the data between the nodes, and the third tier ensures energy efficiency by calculating energy consumption at every level and rotates cluster head among the nodes. The difficult task of energy efficiency is achieved through robust algorithms, which configure the nodes and train the network using a machine learning technique. The simulation results show smooth functioning of the network with less energy consumption. The proposed scheme performs better than Anonymous Authentication for Wireless sensor network in terms of computational overhead, energy consumption, throughput and data drop rate. The usual practice for monitoring in WSNs is to have an event triggered design, i.e., communication between the sensor nodes ( $\mathrm{SNs}$ and MNs) and the sink node (GN) is required only if an anomaly occurs or the server initiates a query. Although this approach saves energy, but has a danger of missing trends. For this reason, in the proposed system design, in addition to an event triggered reporting, attributes readings are also periodically reported to the GN, where event detection and identification algorithm continuously looks for global anomalies.

LEACH is one of the most popular distributed single-hop clustering protocols. In this protocol, the clusters are formed, based on received signal strength. The role of cluster head is periodically rotated amongst the sensor nodes present in the cluster to ensure balanced energy consumption of sensor nodes. This algorithm becomes very inefficient in case of large area sensor networks due to single hop communication of cluster heads to the sink. In this paper, we propose an energy efficient protocol consisting of clustering, cluster head selection/rotation and data routing method to prolong the lifetime of sensor network. In proposed protocol, clusters are formed only once during the lifetime of sensor network, which results in substantial saving of energy.

In order to divide the computation overhead over the network we have proposed clusters of closely related nodes based on the distance and signal strength. Each cluster is headed by a Cluster Head $(\mathrm{CH})$ which takes most of the computation and stores the configuration file of all the nodes. When energy consumption goes beyond the set limit the cluster head configuration is sent to another node. This divides the overhead and maintains energy efficiency. Trust evaluation of a node is carried out based on misbehaviour types. Apart from defining misbehaviour types for direct trust evaluation, we have ranked each type based on level of severity. The types of misbehaviour that we have considered are as follows:

- $\quad$ If a node does not forward messages (Level 5).

- If a node advertises many paths and the paths are declared good (Level 4).

- If a node re-routes to avoid a broken link. However, no errors are observed (Level 3).

- If a node frequently updates routes which is deemed unusual (Level 2).

- If a node tampers with the message header and makes a silent route change (Level 1).

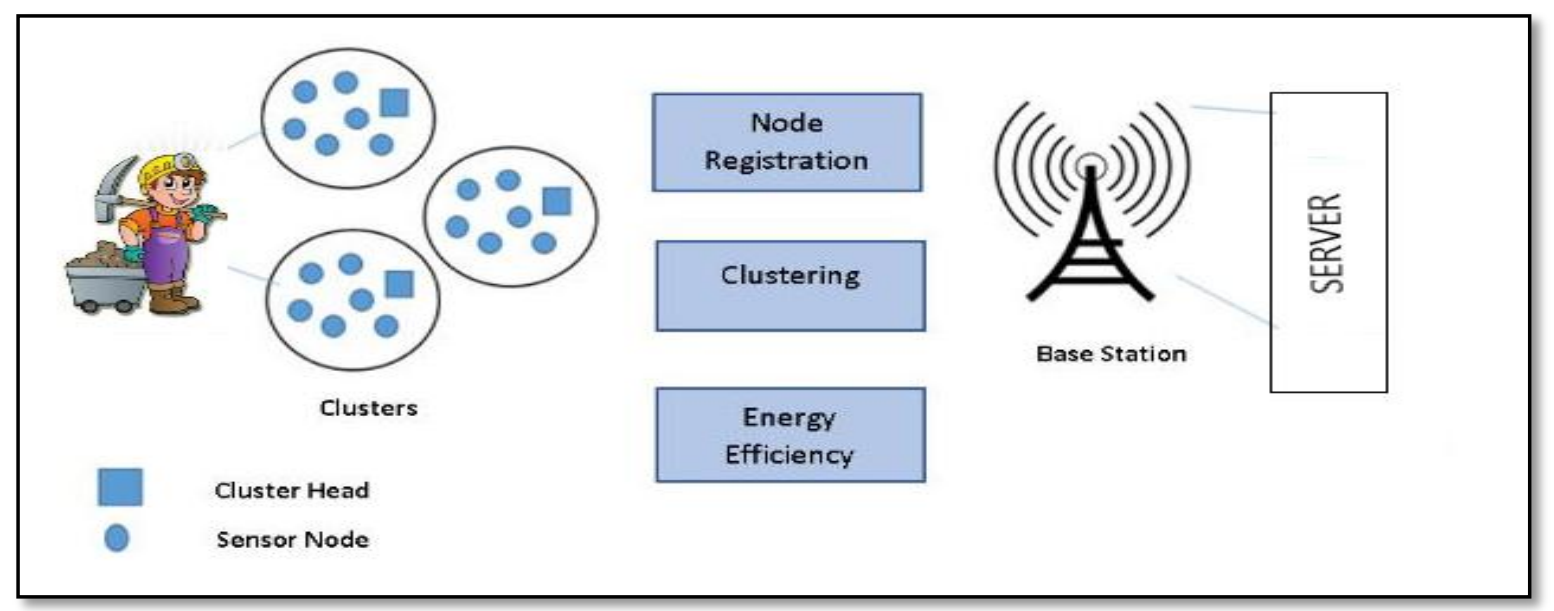

Fig.6. Proposed System

Based on these defined levels of severity, we assume that a node cannot misbehave on two events at the same time. Using these levels of severity, a trust value is assigned to every node. It is important to mention that in proposed scheme, the level of severity is inversely related to the trust value that is if a node misbehaves at a level 1, the node will be assigned the maximum trust value. However, if a node acts on a level 5 severity, then that node will be assigned a low trust value. Depending on the history of information and the time of verification a node through machine learning capability can determine whether the node is malicious or not. The role of cluster head in a cluster must be rotated regularly amongst the sensor nodes to prolong the life time of sensor network by balancing the energy consumption of 
various sensor nodes. Since, cluster head is required to perform extra task of data gathering and data relaying, compared to the regular sensor nodes, its energy drains out faster. Therefore, some mechanism must be adopted to rotate the role of cluster head. The rotation mechanism must ensure balanced energy consumption of all the sensor nodes in cluster. For example, if a node Cluster head $\mathrm{CHa}$ is trying to verify the authenticity of another node $\mathrm{CHb}$, then the $\mathrm{CHa}$ would first check the time at which the information provided by $\mathrm{CHb}$ was last verified. If the information of verification was around for few more successful communications, then the $\mathrm{CHb}$ node would be considered a trusted node and will be assigned a high trust value accordingly. However, in other case the $\mathrm{CHb}$ would be declared as a possible malicious node and would be assigned a low trust value.

Energy efficiency is the overall requirement and the proposed algorithm keeps on calculating it and when energy consumption reaches the defined limit a new $\mathrm{CH}$ is assigned. New $\mathrm{CH}$ assignment depends upon the trust value of the node. In order to become a $\mathrm{CH}$ the node has to achieve a certain level of trust. This is achieved by ranking of rating by other nodes and assignment of lower and upper weight limits depending on a successful communication log.

\section{RESULT}

The result of proposed system can be divided into two parts.

\section{Event Detection and Reporting}

The system consists of three nodes, where the mobile node is carried by the miners will collect the information from the surroundings of miner. The temperature and humidity of surroundings of miner will be collected and send to the stationary node. The stationary nodes placed in the mine environment will also collect the information of toxic gases such as carbon monoxide, methane, vibrations (roof fall) and checks the temperature level. This information will be given to the gateway node. If the information collected is above the certain limit, the alert will be given to the miners. So, the event occurring in the mine environment is detected and reported. These sensor nodes continuously update configurations and trust level and it avoids being malicious by using cluster head rotation algorithm. This will records the initial configurations and saves it in separate file in encrypted form which is used for energy efficient communications between nodes and server.

\section{Simulation}

This paper uses the cluster head rotation algorithm and proposed system having better data gathering, energy efficiency, throughput, etc. compared to other protocols. This proposed algorithm is implemented and simulated in NS2 Simulation shows the proposed algorithm performance throughput is better than the existing system. We use 100 sensor nodes and cluster head in the cluster network and collects the data using clustering algorithms and finally the data reaches the base station. This network simulator simulates the implemented code in an NAM and produces the X-graph as outputs and the output calculates the throughput of the network. Fig. 7 shows the selection of cluster head using NS2 simulation. So, the proposed system uses a LEACH protocol for the formation of cluster and an energy efficient algorithm is used for the rotation of cluster head rotation. Each cluster is headed by a cluster head $(\mathrm{CH})$ which takes most of the computation and stores the configuration file of all the nodes. Cluster heads are changed continuously to divide network energy computation overhead among the nodes. When energy consumptions goes beyond the set limit the cluster head configurations is sent to another node. This divides the overhead and maintains energy efficiency.

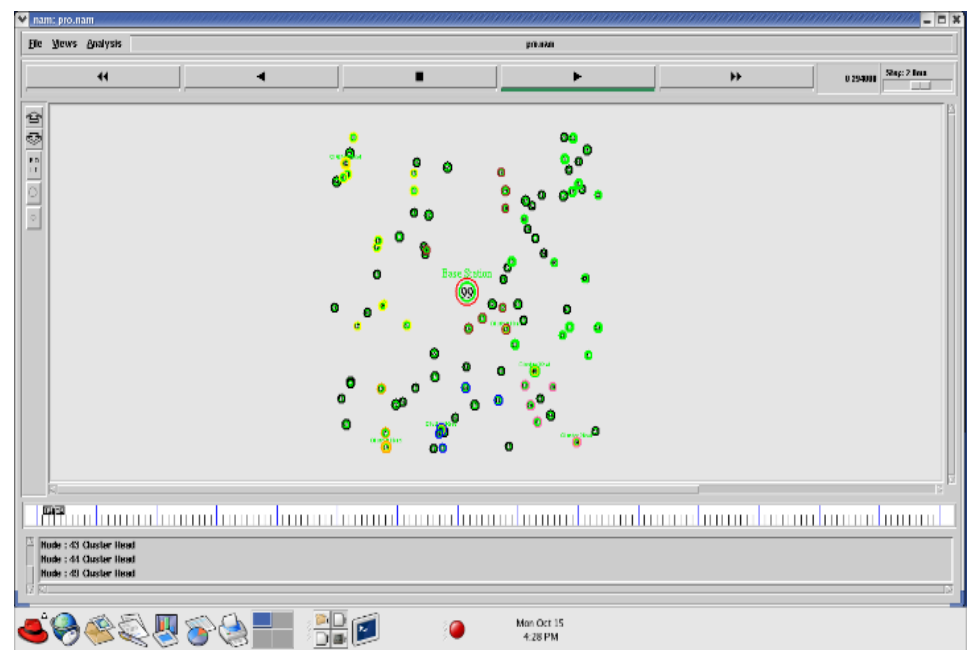

Fig.7. Cluster Head Selection 
So, the Fig. 8 shows the changing of cluster head to other sensor nodes in the underground mine environment. Depending on the history of information and the time of verification a node through machine learning capability can determine whether the node is malicious or not. If the information of verification was around for few more successful communications, then the cluster head node would be considered a trusted node and will be assigned a high trust value accordingly.

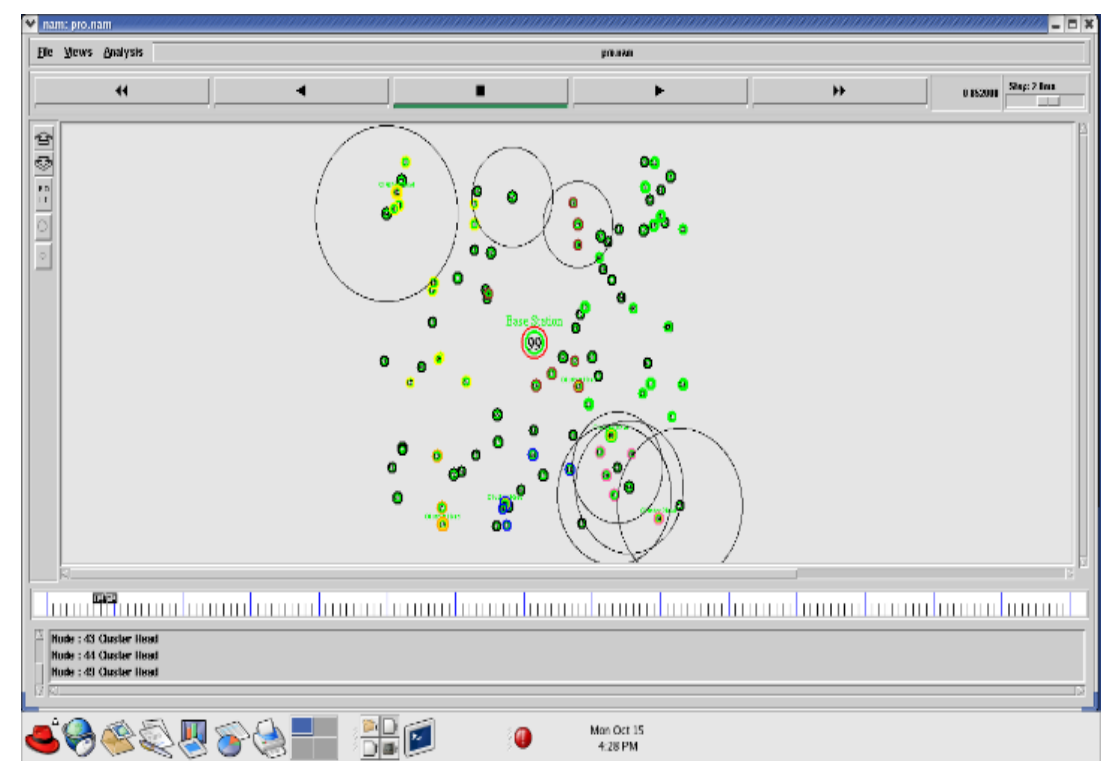

Fig.8. Cluster Head Rotation

Fig. 8 shows the end to end delay of the proposed system and the other algorithm. And it shows clearly that the end to end delay of proposed system is less when compared to other algorithms. That means the proposed system is fast and energy efficient. This achieves a security process and efficient system. And this also shows that the proposed system is energy efficient.

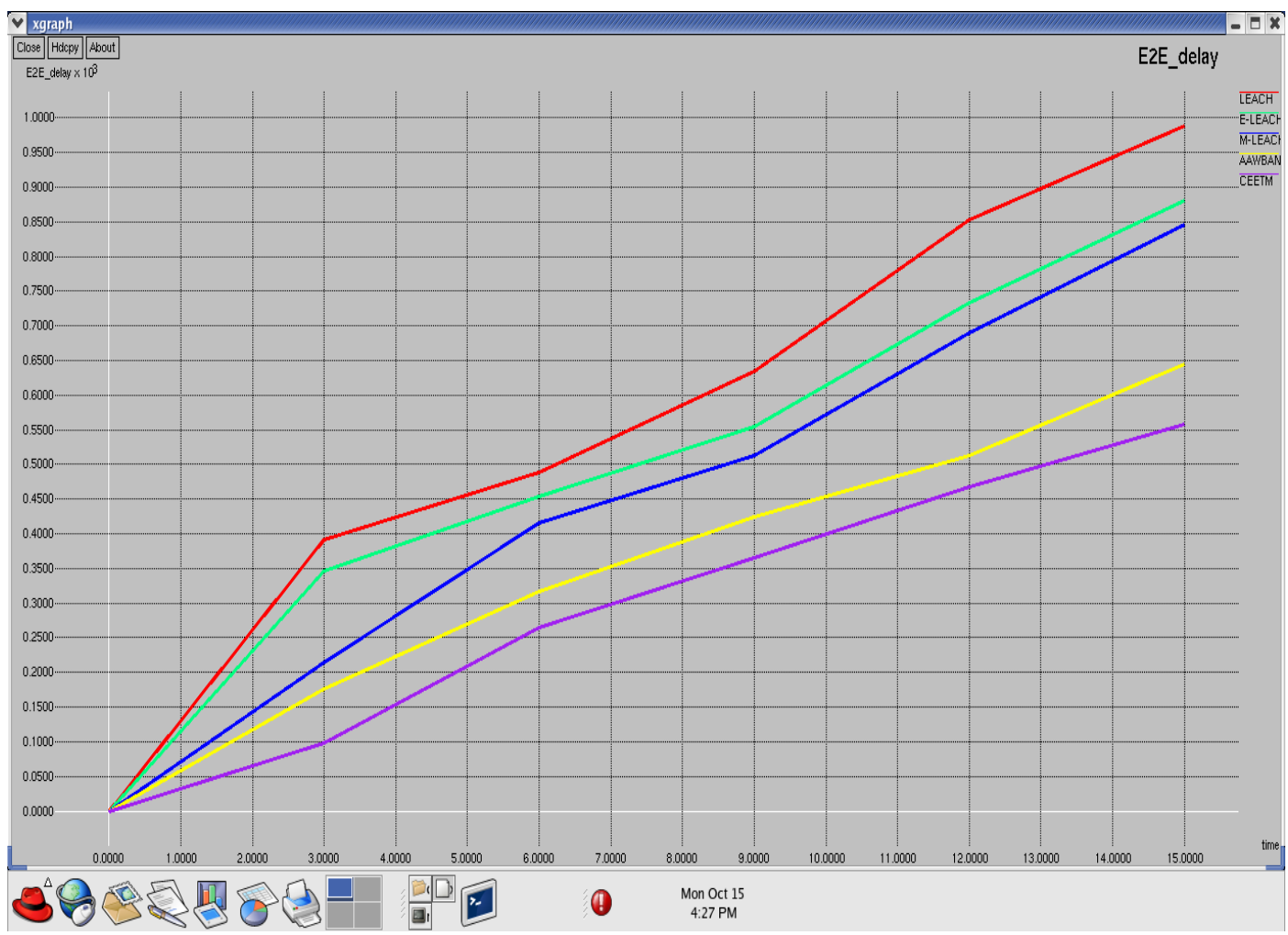

Fig.9. End to End Delay 


\section{CONCLUSION}

In this paper, we propose an energy efficient wireless sensor network with the cluster head rotation algorithm for continuous monitoring and event reporting in underground mine environment. Energy efficiency is the overall requirement and the proposed algorithm keeps on calculating it and when energy consumption reaches the defined limit a new $\mathrm{CH}$ is assigned. New $\mathrm{CH}$ assignment depends upon the trust value of the node. In order to become a $\mathrm{CH}$, the node has to achieve a certain level of trust. This is achieved by ranking of rating by other nodes and assignment of lower and upper weight limits depending on a successful communication log. This system generates reliable disaster detection using sensing data from all over the mine and it reports on integrated system working, both functional and technical performance, in a real-world underground mine environment. This scheme attempts to provide the security and delivery of the data to base station along with stability and better network lifetime.

\section{FUTURE WORK}

In future we can use additional sensors all possible safety issues could be monitored such as gases, dust etc. Zigbee can also be used for the surveillance of mining operations such as subsidence, water leakage etc. The other important data can be communicated through this system making it feasible where wired communication is a hindrance.

\section{REFERENCES}

[1]. H. Safety, Mine and Administration, Accident/illness Investigations Procedures (ph11-i-1) (Maha Handbook Series), U.S. Dept. Labor, Washington, DC, USA, pp. 55-58, 2011.

[2]. K. Kowalski-Trakofler et al., "Underground coal mining disasters and fatalities, United States, 1900-2006," Morbidity Mortality Weekly Rep., vol. 57, no. 51, pp. 1379-1383, 2009.

[3]. J. Shen et al., "A novel routing protocol providing good transmission reliability in underwater sensor networks," J. Internet Technol., vol. 16, no. 1, pp. 171-178, 2015.

[4]. M. Li and Y. Liu, "Underground coal mine monitoring with wireless sensor networks," ACM Trans. Sensor Netw., vol. 5, no. 2, 2009, Art. no. 10 .

[5]. I. O. Osunmakinde, "Towards safety from toxic gases in underground mines using wireless sensor networks and ambient intelligence," Int. J. Distrib. Sensor Netw., vol. 2013, 2013, Art. no. 159273.

[6]. P. Misra, S. Kanhere, D. Ostry, and S. Jha, "Safety assurance and rescue communication systems in high-stress environments: A mining case study," IEEE Commun. Mag., vol. 48, no. 4, pp. 66-73, Apr. 2010

[7]. S. Yarkan, S. Guzelgoz, H. Arslan, and R. R. Murphy, "Underground mine communications: A survey," IEEE Commun. Surveys Tuts., vol. 11, no. 3, pp. 125-142, Jul.-Sep. 2009

[8]. N. Pogkas, G. E. Karastergios, C. P. Antonopoulos, S. Koubias, and G. Papadopoulos, "Architecture design and implementation of an ad-hoc network for disaster relief operations," IEEE Trans. Ind. Informat., vol.3, no. 1, pp. 63-72, Feb. 2007.

[9]. C. Bo et al., "Web of things-based remote monitoring system for coal mine safety using wireless sensor network," Int. J. Distrib. Sensor Netw., vol. 2014, 2014, Art. no. 323127.

[10]. Y. Zhang et al., "An integrated environment monitoring system for underground coal mines wireless sensor network subsystem with multiparameter monitoring," Sensors, vol. 14, no. 7, pp. 13149-13170, 2014.

[11]. X. Niu et al., "The design and evaluation of a wireless sensor network for mine safety monitoring," in Proc. IEEE Global Telecommun. Conf., 2007, pp. 1291-1295

[12]. W. Yang and Y. Huang, "Wireless sensor network-based coal mine wireless and integrated security monitoring information system," in Proc. IEEE Int. Conf. Netw., 2007, pp. 13.

[13]. X. Niu et al., "The design and evaluation of a wireless sensor network for mine safety monitoring," in Proc. IEEE Global Telecommun. Conf., 2007, pp. 1291-1295.

[14]. W. Yang and Y. Huang, "Wireless sensor network-based coal mine wireless and integrated security monitoring information system," in Proc. IEEE Int. Conf. Netw., 2007, pp. 13. 